\title{
Predicting the high strain rate response of plasticised poly(vinyl chloride) using a fractional derivative model
}

\author{
${\text { Akash } \text { Trivedi }^{1, *} \text { and Clive Siviour }}^{1}$ \\ ${ }^{1}$ Department of Engineering Science, University of Oxford, Parks Road, OX1 3PJ, UK
}

\begin{abstract}
Polymers are frequently used in fields as diverse as aerospace, biomedicine, automotive and industrial vibration damping, where they are often subjected to high strain rate or impact loading. Poly(vinyl chloride) (PVC), and its plasticised variants (PPVC), are just two examples of this broad category of materials. Since many polymers exhibit strong rate and temperature dependence, including a low temperature brittle transition, it is extremely important to understand their mechanical responses over a wide range of loading conditions. PVC with $60 \mathrm{wt} \%$ plasticiser is used in this study, as its highly rubbery nature lends itself well to being used in various load mitigation and energy absorption applications. It is challenging to obtain high strain rate data on rubbery materials using conventional techniques such as the split-Hopkinson (Kolsky) bar. Therefore, alternative approaches are required. Based on previous work developing a framework to predict high rate response using a fractional derivative model, Dynamic Mechanical Analysis (DMA) experiments are conducted on the PPVC to construct a master curve of storage modulus. These data are used to part-calibrate a modified Mulliken-Boyce model which also takes into account specimen heating to derive stress-strain relationships at strain rates varying from $0.001 \mathrm{~s}^{-1}$ to $13500 \mathrm{~s}^{-1}$. This model is further calibrated against experiments conducted in a previous study and shown to provide an excellent description of the behaviour at these rates.
\end{abstract}

\section{Introduction}

Poly(vinyl chloride) (PVC) is an example of a widely used amorphous polymer. For many engineering applications, to enhance its ductility and energy absorption, a plasticiser based on a phthalate ester compound, such as diisononyl phthalate (DINP) is added to the PVC [1]. This plasticised PVC (PPVC) exhibits strong rate and temperature dependence in its mechanical properties, including the modulus, yield strength and post-yield behaviour. This sensitivity increases with rate due to inhibitions of the secondary $(\beta)$ transitions [2, 3]; however, characterising the mechanical properties at high strain rates becomes very challenging. Conventional techniques such as the split-Hopkinson (Kolsky) bar do not give accurate measurements of the modulus due to experimental artifacts. In particular, the low specimen stiffness and wavespeed means that it takes significant time to achieve the static equilibrium prerequisite for analysis, whilst low strengths lead to poor signal to noise ratio $[4,5]$. For this reason, a modelling framework has been developed, building on previous research [6, 7], to predict the high rate mechanical response of the PPVC via constitutive models calibrated with data obtained accurately at low strain rates and amplitudes [8].

\section{Material}

The material used in this study is a PVC manufactured by Solvay under the trade name SolVin 264PC with a $60 \mathrm{wt} \%$

\footnotetext{
*e-mail: akash.trivedi@eng.ox.ac.uk
}

of DINP plasticiser. There were two major reasons for this material selection: it is highly plasticised, and so is one of the more challenging to experimentally test at high strain rates; and it allows the validation of the modelling framework since an extensive dataset of experimental results for this material already exists [8]. Another benefit of this particular material is that the response is dominated by hyperelasticity at the lowest strain rates, and that there are no complex interconnections between the primary $(\alpha)$ and secondary $(\beta)$ transitions at the temperatures and rates of interest. A final benefit is that further materials with lower $w t \%$ of plasticiser are available, allowing these challenges to be introduced in future research.

\section{Experiments}

The experimental regime followed in this study was based on existing results from uniaxial compression tests at various strain rates, a Dynamic Mechanical Analysis (DMA) to understand the rate-temperature dependence, and Differential Scanning Calorimetry (DSC) experiments to elucidate the temperature dependence of the heat capacity in the PPVC. Brief descriptions of the experimental methods are provided below, but more details for the DMA and uniaxial experiments can be found in [8].

\subsection{Dynamic Mechanical Analysis (DMA)}

DMA experiments were performed on a TA Instruments Q800 to quantify the "rate" and temperature dependence 
of the primary $\alpha$ transition. Rectangular specimens of dimensions $2.5 \mathrm{~mm} \times 10 \mathrm{~mm} \times 35 \mathrm{~mm}$ were tested in the dual cantilever configuration with a fixed strain amplitude of $0.1 \%$, a temperature range from -80 to $80{ }^{\circ} \mathrm{C}$, and frequencies of $0.5,2,5$, and $10 \mathrm{~Hz}$. Results from the DMA experiments for the storage modulus and the loss modulus are shown in Figure 1a and Figure 1b respectively. For the purpose of comparing the DMA frequency to the strain rate of the uniaxial compression tests, a conversion was made using Equation 1. This assumes that a linear rise in strain occurs in the DMA oscillations over a quarter cycle to the maximum strain amplitude such that the average strain rate can be simply approximated.

$$
\dot{\varepsilon} \approx \frac{\Delta \varepsilon}{\Delta t}=\frac{\varepsilon_{0}}{1 / 4 f}=4 f \varepsilon_{0}
$$

(a)
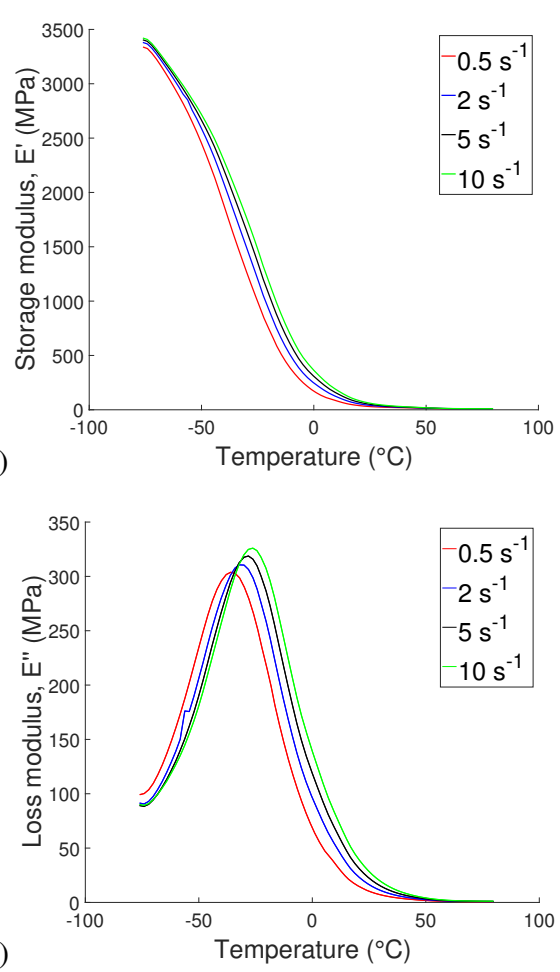

Fig. 1. (a) Dynamic Mechanical Analysis (DMA) results showing the storage modulus and (b) the loss modulus for the PPVC at four discrete rates and varying temperature range.

\subsection{Uniaxial compression}

Uniaxial compression experiments were performed at low rates on an Instron 5980 electromechanical static testing machine, and at high rates on an in-house split-Hopkinson (Kolsky) bar. Intermediate rate characterisation was also performed on a hydraulic press, but these tests are not featured in the current study. The full series of experiments at varying rates and temperatures are presented in [8]. In this study, three distinct rates, Figure 2a, were selected to develop the model.

\subsection{Differential Scanning Calorimetry (DSC)}

At strain rates higher than around $10^{-1} \mathrm{~s}^{-1}$, the compression is no longer isothermal but also features adiabatic heating, as the timescale of heat diffusion out of the specimen is significant compared to the duration of the experiment. In order to calculate the temperature rise, DSC experiments were performed. Here, the heat flow required to change the temperature of a specimen of a given mass is measured and, by comparing to a reference, the heat capacity can be obtained. The results of three tests and the average temperature dependence of the heat capacity is presented in Figure 2b. As expected, as the specimen heats up through the $\alpha$ transition, the free volume increases and density decreases, thereby increasing the heat capacity. A simple quadratic fit, valid in the temperature domain of $\left[-80{ }^{\circ} \mathrm{C}, 180{ }^{\circ} \mathrm{C}\right]$, is also shown in Figure $2 \mathrm{~b}$. The small bump seen between $55-80^{\circ} \mathrm{C}$ is thought to be associated with a small volatile loss during the experiments and not a major transition as a significant change in modulus is not seen in the DMA results.

If it is assumed that all the work generated in straining the material is converted to heat, and the process is fully adiabatic, the resultant temperature rise in the specimen can be calculated using Equation 2. The temperature rise for the two relevant cases where adiabatic heating is experienced is shown in Figure 2c.

$$
\Delta T \approx \frac{1}{\rho C_{p}(T)} \int_{0}^{\varepsilon} \sigma(\varepsilon) d \varepsilon
$$

\section{Constitutive Modelling}

\subsection{Background}

\subsubsection{Hyperelasticity}

For many polymers that exhibit large elasticity, a linear elastic model like that of Hooke cannot capture the mechanical behaviour. Thus, a non linear model of this hyperelasticity is needed. Many models have been proposed since the early invariant based continuum Mooney-Rivlin model [9, 10]. Two popular approaches are the stretch based continuum Ogden model [11] and the Langevin chain statistic based macromolecular Arruda-Boyce model $[12,13]$. These models are good at capturing large stretch dominated elasticity, however fail at capturing rate dependence or plasticity. Therefore, suitable adaptations to the models must be made to incorporate these features in the material response.

\subsubsection{Viscoelasticty}

The rate and temperature dependence of many materials has been known for some time [14, 15], however modelling this can be challenging. From a DMA experiment it is possible to see the extent to which the modulus of a material can vary as it passes through its transitions because of either temperature or loading rate. Rate dependence 
(a)

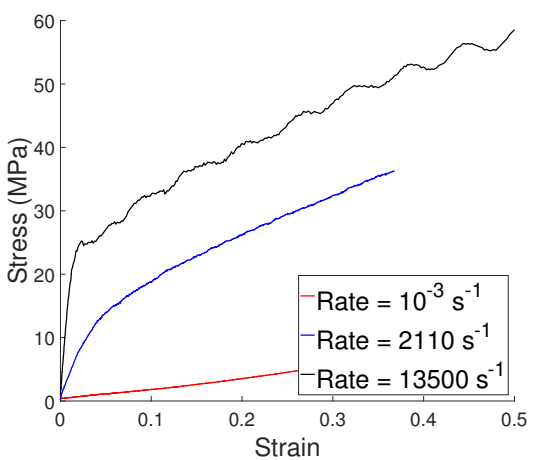

(b)
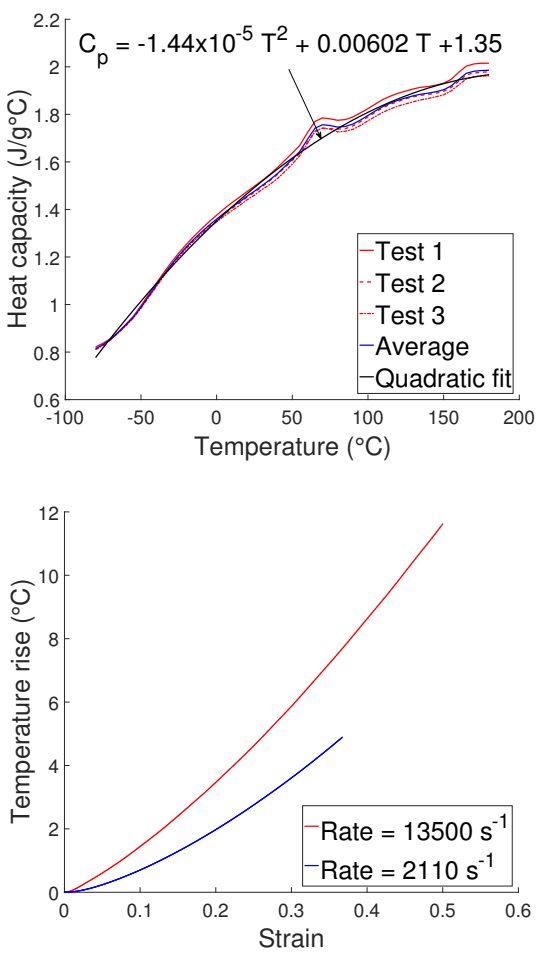

Fig. 2. (a) Stress strain curves from the uniaxial compression experiments [8], (b) data from DSC experiment on obtaining the temperature dependence of the heat capacity, $C_{p}$, for the PPVC, and (c) the temperature rise due to adiabatic heating experienced in the two high strain rate cases.

must form a central part of any polymer model. Linear viscoelastic models have been widely used with springs and dashpots as either Maxwell or Kelvin-Voigt elements, in parallel or series with a spring, forming a Standard Linear Solid or Zener model [16], or in the generalised case, a Prony series with numerous elements $[17,18]$. However, this approach is not wholly satisfactory and a further generalisation can be made if fractional derivatives are used [19-21] to introduce non linear viscoelastic behaviour that is more representative of real materials.

\subsubsection{Plasticity}

Once the stretch exceeds the elastic limit, plasticity can no longer be neglected. Rate dependent plasticity has been implemented previously with the Mulliken-Boyce model
$[22,23]$, including the effects of thermal softening through adiabatic heating by explicitly considering polymer transitions [24]. However, the interconnection between the rate, temperature and the seamless integration of adiabatic heating into a model with predictive capabilities at high strain rates has not been fully explored and is therefore the focus of this study.

\subsection{Enhanced Fractional Thermo-Visco-Hyperelasto-Plastic (FTVHEP) model}

The premise of the enhanced FTVHEP modelling framework is as follows. DMA experiments at low strain rates and amplitudes can be conducted to find the ratetemperature dependence of the material's storage and loss modulus. The in-phase storage modulus can be used as a proxy for the initial modulus in the stress strain response of the specimen for any given starting temperature and strain rate (based on Equation 1). Adiabatic heating through the test increases the temperature, and this temperature increase can be reflected with a shift in the DMA master curve to a different higher reference temperature for each time step. The relaxation modulus can thus be effectively obtained with dependences on time and temperature accurately represented. This varying modulus can be incorporated directly into the conventional MullikenBoyce model to predict the full stress strain response at varying rates. This represents an improvement to the existing model since the enhanced model takes into account the time-temperature dependence on the modulus as the experiment progresses.

Thermal softening from adiabatic heating in the specimen at high strain rates has previously been experimentally simulated $[25,26]$ but efforts to integrate the effects into an acceptable model have had limited success. In Section 3.3, Figure $2 \mathrm{c}$ shows the temperature rise due to adiabatic obtained based on previous experiments. Based on the empirical shift factors derived from the DMA experiments, Figure $3 b$, the shift in the master curve required for the given temperature rise can be calculated. The master curve for the storage modulus traditionally has been represented with a Prony fit as described in Equation 3, where $E_{\infty}$ is the long term modulus, $E_{i}$ is the modulus for an individual element, and $t_{i}$ is the relaxation time for that element (this is prescribed as $t_{1}=10^{-18}$ for $E_{1}, t_{2}=10^{-17}$ for $E_{2}$, and so on based on where the individual elemental transition is centred up to $t_{22}=10^{3}$ for $E_{22}$ ).

$$
E^{*}=E^{\prime}+i E^{\prime \prime}=E_{\infty}+\sum_{i=1}^{N}\left[E_{i} \frac{i f}{i f+t_{i}^{-1}}\right]
$$

However, to achieve a successful fit over the 22 decades represented in the current data, Figure $3 \mathrm{a}$, convention dictates 22 elements in the Prony series plus a term for the long term modulus. The advantage of using a Fractional model, see Equation 4, is that realistic history dependent non linear viscoelasticity in the storage modulus can be represented with minimal terms (Table 1). Here, 


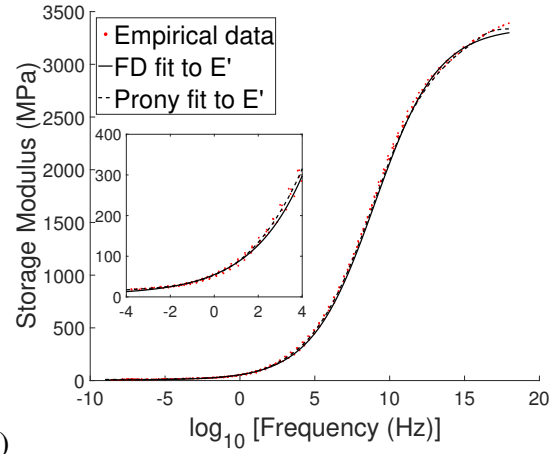

(a)

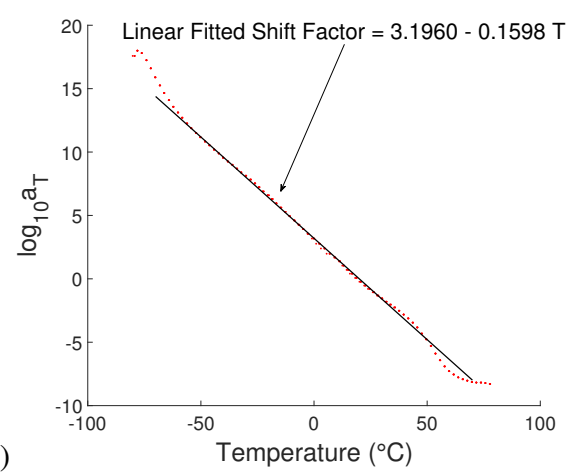

Fig. 3. (a) Fitting traditional Prony series and FD models to the DMA master curve and (b) empirical shift factors obtained from constructing the master curve.

$E_{0}$ refers to the initial modulus when the specimen has no time to relax, $E_{\infty}$ is as before, and $t_{1}$ is the model relaxation time corresponding to where the transition is centred. In the Fractional model, $\beta$ is the fractional power that dictates the slope of the transition in the storage modulus DMA master curve. Physically, when $\beta=1$ it represents a Newtonian fluid, and when $\beta=0$, a linear elastic body.

$$
\begin{aligned}
E^{*} & =E^{\prime}+i E^{\prime \prime} \\
& =\frac{E_{0}}{1+E_{0} / E_{\infty}}\left[\frac{1+\left(1+E_{0} / E_{\infty}\right)\left(i f t_{1}\right)^{\beta}}{1+\left(i f t_{1}\right)^{\beta}}\right]
\end{aligned}
$$

Once the master curve for the storage modulus is shifted accordingly with the knowledge of the temperature rise due to adiabatic effects, this new time-temperature dependent representation of the relaxation modulus can be directly replaced with modulus term in the existing Mulliken-Boyce model. The improvement in the model means that instead of using a single value of the modulus, a continually relaxing modulus with integrated thermal softening is used to better capture large strain mechanical response at high strain rates.

The stress strain curves based on the enhanced model are compared to the conventional Mulliken-Boyce model in Figure 4 for two high strain rate cases. The Langevin hyperelasticity is based on the rubbery modulus, $E_{\infty}(=$ $C_{R}$ ), obtained directly from the DMA experiment, and the limiting chain extensibility, $\sqrt{N}$, obtained through a least squares curve fit against the lowest strain rate experiments shown in Figure 4a. The rest of the parameters for the enhanced model are obtained as shown in Table 2, by either calibrating against the $\dot{\varepsilon}=2110 \mathrm{~s}^{-1}$ case shown in Figure $4 \mathrm{~b}$ or by using yield data from [8]. Parameters associated with the preferred athermal shear strength state and softening slope are yet to be fully established and will be studied in more detail with varying temperature data from [8]. The model is then part validated against the highest strain rate test in the study shown in Figure 4c. Both high rate cases exhibit the success of using this enhanced FTVHEP modelling framework over the conventional Mulliken-Boyce model.

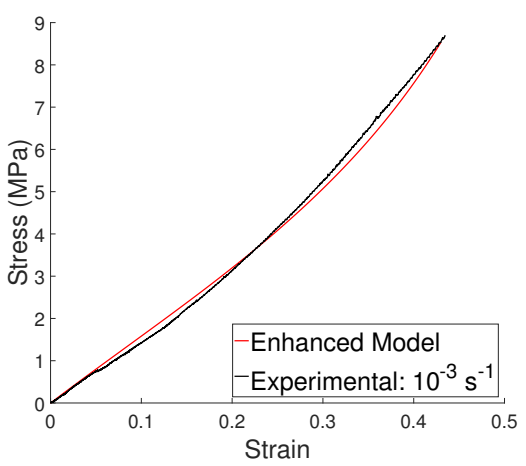

(a)

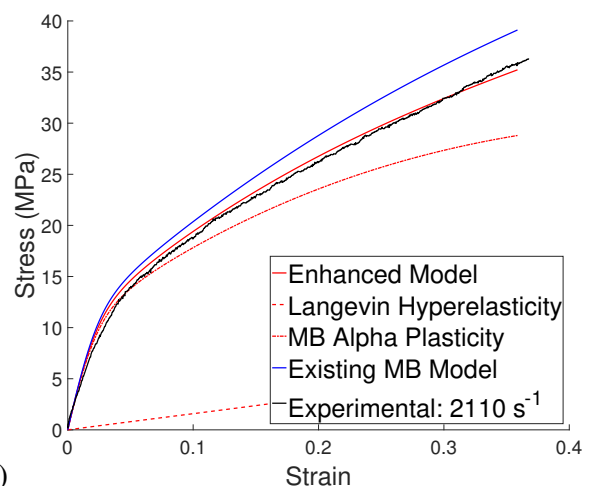

(b)

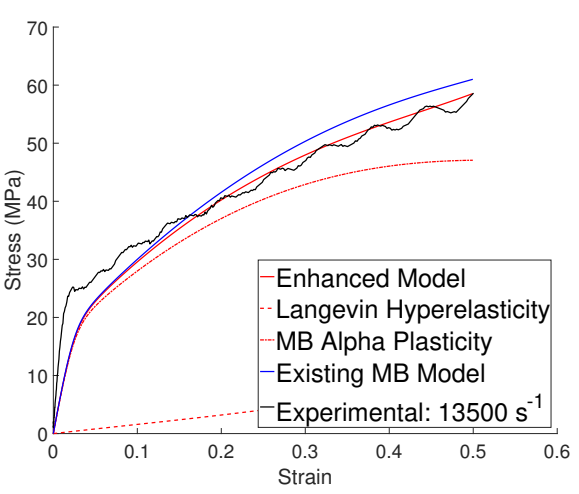

Fig. 4. Comparing the FTVHEP modelling framework to the conventional Mulliken-Boyce model at strain rates: (a) $\dot{\varepsilon}=$ $10^{-3} \mathrm{~s}^{-1}$, (b) $\dot{\varepsilon}=2110 \mathrm{~s}^{-1}$, and (c) $\dot{\varepsilon}=13500 \mathrm{~s}^{-1}$.

\section{Conclusions}

In this study, the development of an enhanced FTVHEP modelling framework based on the Mulliken-Boyce model has been presented. This model provides significant improvements to the conventional Mulliken-Boyce in that it offers seamless integration of adiabatic heating during ex- 
Table 1. Parameters used for the Prony and Fractional fits to the DMA data.

\begin{tabular}{|c|c|c|c|c|c|c|c|c|c|c|c|c|}
\hline \multirow{4}{*}{$\begin{array}{c}\text { Prony series } \\
\text { (MPa) }\end{array}$} & $E_{\infty}$ & $E_{1}$ & $E_{2}$ & $E_{3}$ & $E_{4}$ & $E_{5}$ & $E_{6}$ & $E_{7}$ & $E_{8}$ & $E_{9}$ & $E_{10}$ & $E_{11}$ \\
\hline & 10.2 & 141 & 85.7 & 133 & 156 & 220 & 286 & 371 & 407 & 388 & 328 & 254 \\
\hline & $E_{12}$ & $E_{13}$ & $E_{14}$ & $E_{15}$ & $E_{16}$ & $E_{17}$ & $E_{18}$ & $E_{19}$ & $E_{20}$ & $E_{21}$ & $E_{22}$ & \\
\hline & 192 & 122 & 87.4 & 65.3 & 37.2 & 23.0 & 12.7 & 6.18 & 4.12 & 1.27 & 7.04 & \\
\hline
\end{tabular}

\begin{tabular}{|c|c|c|c|c|}
\hline $\begin{array}{c}\text { Fractional } \\
\text { model }\end{array}$ & $E_{0}(\mathrm{MPa})$ & $E_{\infty}(\mathrm{MPa})$ & $\beta$ & $t_{1}(\mathrm{~s})$ \\
\cline { 2 - 5 } & 3350 & 2.5 & 0.2 & $10^{-9}$ \\
\hline
\end{tabular}

Table 2. Parameters for the enhanced FTVHEP modelling framework and how they were obtained.

\begin{tabular}{|c|c|}
\hline \multicolumn{2}{|c|}{ Parameters for the Mulliken-Boyce based FTVHEP modelling framework } \\
\hline Poisson's ratio, $v=0.5$ & Incompressibility assumption \\
\hline Shear modulus, $\mu_{0}=1.12 \mathrm{GPa}$ & Calculated based on $v$ and $E_{0}$ \\
\hline Athermal shear strength, $S_{0}=172 \mathrm{MPa}$ & Calculated based on $\mu_{0}$ \\
\hline Absolute temperature, $\theta=293 \mathrm{~K}$ plus temperature rise & Test value plus rise from Figure 2c \\
\hline Limiting chain extensibility, $\sqrt{N}=1.52$ & Fit to lowest strain rate data in [8] \\
\hline Long term (rubbery) modulus, $E_{\infty}\left(=C_{R}\right)=2.5 \mathrm{MPa}$ & From DMA experiments \\
\hline Pressure coefficient, $\alpha_{p}=0.22$ & From [27] \\
\hline Pre-exponential factor, $\dot{\gamma_{0}}=8 \times 10^{23} \mathrm{~s}^{-1}$ & From yield data in [8] \\
\hline Activation energy, $\Delta G=1.98 \times 10^{-19} \mathrm{~J}$ & From yield data in [8] \\
\hline Softening slope, $h=1 \mathrm{GPa}$ & Fit to $\dot{\varepsilon}=2110 \mathrm{~s}^{-1}$ data \\
\hline Preferred athermal shear strength state, $S_{s s}=10 \mathrm{GPa}$ & Fit to $\dot{\varepsilon}=2110 \mathrm{~s}^{-1}$ data \\
\hline Boltzmann constant, $k_{B}=1.38 \times 10^{-23} \mathrm{~m}^{2} \mathrm{~kg} \mathrm{~s}^{-2} \mathrm{~K}^{-1}$ & Physical constant \\
\hline
\end{tabular}

perimentation to be able to predict the full stress strain response at rates up to the order of $10^{4} \mathrm{~s}^{-1}$.

Despite modelling successes, there is work yet to be done to fully validate the predictive framework using only quasi-static experiments. Currently, the adiabatic heating, pre-exponential factor, activation energy, softening slope, and the preferred state rely on high rate data. Furthermore, the FTVHEP modelling framework is yet to be validated against less plasticised PVC where the interplay between the $\alpha$ and $\beta$ transitions is significant. However, this framework provides a promising way forward for both experimentalists and modellers of soft materials at high strain rates.

This material is based upon work supported by the Air Force Office of Scientific Research, Air Force Material Command, USAF under Award No. FA9550-15-1-0448. Any opinions, findings, and conclusions or recommendations expressed in this publication are those of the author(s) and do not necessarily reflect the views of the Air Force Office of Scientific Research, Air Force Materiel Command, USAF.

\section{References}

1. G. Matthews, PVC: Production, Properties and Uses, Vol. 587 (The Institute of Materials, 1996)

2. S. Walley, J. Field, DYMAT j 1, 211 (1994)

3. C. Bauwens-Crowet, J.C. Bauwens, G. Homès, Journal of Polymer Science 7, 735 (1969)

4. C.R. Siviour, High strain rate characterization of polymers, in AIP Conference Proceedings (2017), 10, pp. $1-12$
5. C.R. Siviour, J.L. Jordan, Journal of Dynamic Behavior of Materials 2, 15 (2016)

6. A.R. Trivedi, C.R. Siviour, AIP Conference Proceedings (Accepted) (2018)

7. A.R. Trivedi, C.R. Siviour, A framework for analyzing hyper-viscoelastic polymers, in Constitutive Models for Rubber X, edited by A. Lion, M. Johlitz (CRC Press, 2017), pp. 529-535

8. M.J. Kendall, C.R. Siviour, Proceedings of the Royal Society A: Mathematical, Physical and Engineering Sciences 470, 20140012 (2014)

9. M. Mooney, Journal of Applied Physics 11, 582 (1940)

10. R.S. Rivlin, Philosophical Transactions of the Royal Society A: Mathematical, Physical and Engineering Sciences 241, 379 (1948)

11. R. Ogden, Proceedings of the Royal Society A: Mathematical, Physical and Engineering Sciences 326, 565 (1972)

12. E.M. Arruda, M.C. Boyce, Journal of the Mechanics and Physics of Solids 41, 389 (1993)

13. E.M. Arruda, M.C. Boyce, R. Jayachandran, Mech. Mater. 19, 193 (1995)

14. A.V. Tobolsky, Journal of the American Chemical Society 74, 3786 (1952)

15. Ferry J.D., Viscoelastic Properties of Polymers (Wiley, 1980), ISBN 9780471048947

16. C.M. Zener, S. Siegel, The Journal of Physical and Colloid Chemistry 53, 1468 (1949)

17. Y.C. Fung, Foundations of solid mechanics (Prentice Hall, 1965)

18. N.W. Tschoegl, The Phenomenological Theory of Linear Viscoelastic Behavior: An Introduction (Springer 
Berlin Heidelberg, 1989)

19. R.C. Koeller, Acta Mechanica 58, 251 (1986)

20. R.L. Bagley, P.J. Torvik, Journal of Rheology 27, 201 (1983)

21. R.L. Bagley, P.J. Torvik, Journal of Rheology 30, 133 (1986)

22. A. Mulliken, M. Boyce, International Journal of Solids and Structures 43, 1331 (2006)

23. J.L. Jordan, J.R. Foley, C.R. Siviour, Mechanics of Time-Dependent Materials 12, 249 (2008)
24. A.D. Mulliken, S.Y. Soong, M.C. Boyce, R.E. Cohen, Journal de Physique IV (Proceedings) 134, 217 (2006)

25. M.J. Kendall, C.R. Siviour, Polymer (United Kingdom) 54, 5058 (2013)

26. J. Furmanski, C.M. Cady, E.N. Brown, Polymer (United Kingdom) 54, 381 (2013)

27. K. Pae, S. Bhateja, Journal of Macromolecular Science-Reviews in Macromolecular Chemistry 13, 1 (1975) 